\title{
On Some Fuzzy Relations for Color Information
}

\author{
D. Sánchez \\ European Centre for Soft Computing \\ Mieres, Asturias, Spain \\ daniel.sanchezf@softcomputing.es
}

\author{
J. M. Soto-Hidalgo \\ Dept. Computer Architecture, \\ Electronics, and Electronic Technology \\ University of Córdoba, Spain \\ jmsoto@uco.es
}

\author{
J. Chamorro-Martínez, P. Martínez-Jiménez \\ Dept. Computer Science and A.I. \\ University of Granada, Spain \\ \{jesus,pedromartinez\}@ decsai.ugr.es
}

\begin{abstract}
In this paper we introduce several fuzzy resemblance relations between different combinations of crisp and fuzzy colors. As a starting point, we discuss about fuzzy colors and fuzzy color spaces as a way to represent color information, providing examples of conjunctive and disjunctive use of fuzzy colors for this purpose. Then we determine different types of relations measuring the degree of matching between color information of different kinds, using well known results from fuzzy sets and possibility theories. We study the particular case in which the user considers there is no resemblance between the fuzzy colors comprising the fuzzy color space under consideration. The proposal is based on definitions of fuzzy color and fuzzy color space provided by the authors in previous works.

Index Terms-Fuzzy Color Information, Fuzzy Relations.
\end{abstract}

\section{INTRODUCTION}

Humans and computers describe colors in a completely different way, a problem that is known as the semantic gap. Whilst computers represent colors as points in some threedimensional (color) space, humans employ a comparatively much smaller collection of linguistic terms like red, light blue, and strong green.

This fact poses very interesting problems when developing applications for managing color information in which the source or destination of part of the information is a human user, whilst the rest has been obtained by means of sensors or has been generated synthetically [1]. This is the case in applications like generating descriptions of pictures for visually impaired people, determining the suitability of a certain soil for a specific cultivation, or detecting potentially cancerogenous areas in the skin, just to mention a few among a huge number.

In this paper, we are interested in problems about uncertainty in both the representation and the relationships between pieces of color information. Several authors have pointed out the suitability of the theory of fuzzy sets for this purpose, leading to the introduction of notions like fuzzy color for representing the compatibility between triplets of real values (crisp colors) and linguistic terms [2]-[12].

However, to the best of our knowledge, no deep study of how to use these concepts for representing uncertain color information, or relating pieces of color information for flexible query answering, have been made available in the literature. Our objective in this work is to go a step forward in this direction.

This work has been partially supported by the Spanish Government under project TIN2009-08296.
The rest of the paper is organized as follows. In section II we describe the approach to fuzzy colors and fuzzy color spaces introduced in [12]. Possible uses of fuzzy colors for representing color information are described in section III. In section IV we introduce different fuzzy relations for evaluating different types of matching queries involving color information. These relations are particularized to the case of a specific resemblance relation between fuzzy colors in section V. Finally, section VI contains our conclusions and future research plans in this area.

\section{FuZzy Modelling OF COLORS}

All along the paper, we will consider the notions of fuzzy color (section II-A) and fuzzy color space (section II-B) introduced in [12]. We introduce these notions in the following sections.

\section{A. Fuzzy color}

In order to represent the semantic compatibility between crisp colors and linguistic color terms, in [12] we introduce the following definition of fuzzy color:

Definition 2.1: [12] A fuzzy color $\widetilde{C}$ is a normalized fuzzy subset of crisp colors.

As previously explained, crisp colors can be represented as a triplet of real numbers corresponding to coordinates in a color space. Hence, a fuzzy color can be defined as a normalized fuzzy subset of points of a color space. From now on, we shall assume that a generic color space with components $X$, $\mathrm{Y}$ and $\mathrm{Z}$ whose domains are $D_{X}, D_{Y}$ and $D_{Z}$ respectively, is employed. We shall call this color space $X Y Z$ (not to be confused with the CIE XYZ color space, which is just one particular case). This leads to the following more specific definition [12]:

Definition 2.2: [12] A fuzzy color $\widetilde{C}$ is a linguistic label whose semantics is represented in a color space $\mathrm{XYZ}$ by a normalized fuzzy subset of $D_{X} \times D_{Y} \times D_{Z}$.

Def. 2.2 implies that for each fuzzy color $\widetilde{C}$ there is at least one crisp color $\mathbf{r}_{\widetilde{C}}$ such that $\widetilde{C}\left(\mathbf{r}_{\widetilde{C}}\right)=1$. Also, the membership function is not required to be convex. This leaves open the possibility to define color terms like for example chocolat, that may be represented by the union of the fuzzy sets for color 
terms like white, dark brown, and black. Following this idea, we shall consider two kind of fuzzy colors:

- Atomic colors, those colors that are not defined on the basis of other colors, like white, dark brown, and black in the previous example. We shall consider that atomic colors are represented by means of convex fuzzy sets; in particular this implies ${ }^{\alpha} \widetilde{C}$ is a convex volume $\forall \alpha \in(0,1]$, where ${ }^{\alpha} \widetilde{C}$ is the $\alpha$-cut of degree $\alpha$ of the fuzzy color $\widetilde{C}$.

- Derived colors, those defined on the basis of other colors by means of logical operations, like the color chocolat of the previous example.

Since the representation of fuzzy colors is a matter of semantics, we have that not every convex fuzzy subset of colors corresponds necessarily to an atomic fuzzy set. Another important problem related to the semantics arises because it is possible to represent the same fuzzy color by means of different fuzzy subsets on different color spaces, provided they have the same expressive power. For example, a fuzzy color red may be represented as a fuzzy subset with semantics "approximately $[255,0,0]$ " if we employ the RGB color space or as a fuzzy subset with semantics "approximately $[0,1,128]$ " if we use the HSV space. However, if we apply a transformation of the crisp colors in "approximately $[255,0,0]$ " to HSV and we maintain the membership degrees, the result may not be "approximately $[0,1,128]$ "; even worst, when performing such transformations from one color space to another, we can obtain a non-convex fuzzy set! Hence, a fuzzy subset of colors that semantically is an atomic fuzzy color has a non-convex representation in other spaces.

A particular case of this problem comes from the fact that the expressive power (the gamut) is not the same for every color space, so it could be the case that some crisp colors with degree 0 appear inside the convex volume when changing the representation, corresponding to colors that the original color space is not able to represent. However, non-convexity on the support of the fuzzy set could in principle arise as well because of the transformation from one color space to another because of a spatial redistribution of crisp colors in the color space, even if both color spaces have the same gamut. As a consequence, the notion of atomic fuzzy color may be dependent on the color space employed in the definition of the membership function. Hence, we introduce the following definitions: let XYZ be a (crisp) color space.

Definition 2.3: [12] A XYZ fuzzy color is a fuzzy color whose membership function is defined on $\mathrm{XYZ}$.

Definition 2.4: [12] An atomic XYZ fuzzy color is a fuzzy color whose membership function is convex on $\mathrm{XYZ}$.

From now on we will say that a fuzzy color is simply atomic when it is atomic in some color space XYZ.

\section{B. Fuzzy color space}

For extending the concept of color space to the case of fuzzy colors, and assuming a fixed color space XYZ, with $D_{X}, D_{Y}$ and $D_{Z}$ being the domains of the corresponding color components, the following definition is introduced in [12]:
Definition 2.5: [12] A fuzzy color space $\widetilde{X Y Z}$ is a set of atomic XYZ fuzzy colors that define a partition of $D_{X} \times D_{Y} \times$ $D_{Z}$.

We consider that a fuzzy partition of the color space $X Y Z$ is a crisp set of $m$ fuzzy colors $\widetilde{C}_{1}, \ldots, \widetilde{C}_{m}$ verifying the following properties [12]:

1) $\bigcup_{\{1, \ldots, m\}} \sup \left(\widetilde{C}_{i}\right)=X Y Z$, i.e., the union of the support of the $\widetilde{C}_{i}$ covers the whole space.

2) $\operatorname{ker}\left(\widetilde{C}_{i}\right) \cap \operatorname{ker}\left(\widetilde{C}_{j}\right)=\emptyset \forall i \neq j$, i.e., the kernels of the $\widetilde{C}_{i}$ and $\widetilde{C}_{j}$ are pairwise disjoint.

3) $\forall i \in\{1, \ldots, m\} \exists \mathbf{c} \in X Y Z$ such that $\widetilde{C}_{i}(\mathbf{c})=1$, i.e., there is at least one object fully representative of the fuzzy color $\widetilde{C}_{i}$.

Condition 3 is always verified by definition of fuzzy color. Condition 1 implies $\forall \mathbf{c} \in X Y Z \exists i \in\{1, \ldots, m\}$ such that $\widetilde{C}_{i}(\mathbf{c})>0$. Conditions 2 and 3 imply $\widetilde{C}_{i} \nsubseteq \widetilde{C}_{j} \forall i \neq j$.

\section{COLOR INFORMATION}

Fuzzy colors allow us to represent color information in a computer, filling the semantic gap with human's perception of color. However, it is important to remark that the same fuzzy color may represent information with different semantics depending on its use.

Fuzzy colors can be used both in a conjunctive and disjunctive way as pieces of information [13]-[16]. An example of a conjunctive use is to answer with a fuzzy color $\widetilde{C}$ to the question "which are the red colors?". In this case, each crisp color $\mathrm{c}$ is part of the answer with a degree given by $\widetilde{C}(c)$. An example of disjunctive use is to provide a fuzzy color for answering the question "what's the color of that car?". In this second case, the true answer is a single crisp color, but we do not know exactly what one, so we answer by giving a restriction on the set of crisp colors given by the fuzzy color $\widetilde{C}$, i.e., the possibility that a certain crisp color $\mathrm{c}$ is the color of the car is $\widetilde{C}(c)$. Hence, a disjunctively-used fuzzy color is a possibility distribution on the set of crisp colors. From now on we shall denote the conjunctive (resp. disjunctive) use of fuzzy colors as $\mathrm{Cu}$ (resp. $\mathrm{Du}$ ), i.e., we shall say that a fuzzy color is conjunctively used $(\mathrm{Cu})$ or disjunctively used $(\mathrm{Du})$.

Hence, in this paper we shall consider that color information may be expressed by means of:

1) A crisp color $c \in X Y Z$, e.g., this pixel has color $[\mathbf{0 , 0 , 0}]$ in $R G B$.

2) A conjunctively-used fuzzy color, e.g., I like red cars; the grass is green. It defines the compatibility degree between a linguistic term and the crisp colors in a crisp color space.

3) A disjunctively-used fuzzy color (possibility distribution on the set of crisp colors), corresponding to a description by means of a flexible restriction of our knowledge about the actual value of a variable whose possible values are crisp colors. For example, my car is red; 
Table I indicates the actual information represented by each of the previous cases, as well as the presence of imprecision and/or uncertainty.

TABLE I

COLOR INFORMATION, TYPE OF VALUE REPRESENTED, AND PRESENCE OF IMPRECISION AND/OR UNCERTAINTY IN THE REPRESENTATION.

\begin{tabular}{llll}
\hline Representation & Value & Imprec. & Uncert. \\
\hline $\mathrm{Crisp}$ & Crisp color & No & No \\
\hline $\mathrm{Cu}$ & Cu Fuzzy Color & Yes & No \\
\hline $\mathrm{Du}$ & Crisp color & No & Yes \\
\hline
\end{tabular}

\section{MATCHING COLOR INFORMATION}

Matching color information is crucial for practical applications involving fuzzy color spaces. In particular, when we are given statements involving color information coming from different sources, the assessment of these statements depends usually in the degree of matching between them. In this section we propose how to calculate the degree of matching associated to certain expressions involving two pieces of color information, depending on the semantics of the color information employed (any of those explained in the previous section).

The basic information to be employed in color information matching is intimately linked to the own definition of a fuzzy color space, since the representation of each fuzzy color is the fuzzy set of crisp colors that are compatible with the fuzzy color. This representation induces a resemblance relation for crisp colors based on the idea that crisp colors appearing to a certain degree in the representation of a fuzzy color are supposed to be resemblant at least to the minimum of those degrees. This notion of resemblance induced by a fuzzy color can be extended to resemblance induced by a fuzzy color space just taking the maximum degree among all the fuzzy colors of the fuzzy color space, as we shall see. We shall denote by $r_{\widetilde{C}}$ and $R_{\widetilde{X Y Z}}$ the resemblance relations induced by $\widetilde{C}$ and $\widetilde{X Y Z}$, respectively. For the sake of simplicity, when there is a single clear fuzzy color space $\widehat{X Y Z}$, we shall denote the relations as $r$ and $R$, respectively.

In addition to defining each fuzzy color, we shall consider that users can provide their perception of resemblance between fuzzy colors by means of a fuzzy resemblance relation (reflexive and symmetric), that we will denote by $S_{\widetilde{X Y Z}}$ (or, again, simply $S$ when the fuzzy color space is known). Notice that $S$ is not expected to be transitive in general, and this is consistent with our view of fuzzy color resemblance. For example, one might say that red and orange are resemblant to some degree, and orange and yellow are resemblant to the same degree, but red and yellow are resemblant to a lesser degree than the former. Let us point out that this resemblance is to be taken into account only when the fuzzy color is used in a conjunctive way (as a whole).

Let us remark that compatibility and resemblance of fuzzy colors are different relations, in particular we assume $S$ to be independent of the representation of fuzzy colors. Hence, there is no problem in considering that two fuzzy colors are compatible to a certain degree $\alpha>0$ (i.e., there is at least one
TABLE II

MOST USUAL CASES WHEN MATCHING PIECES OF COLOR INFORMATION, AND THE CORRESPONDING EQUATION NUMBER.

\begin{tabular}{|c|c|c|c|}
\hline & c' & $\mathrm{Cu} \widetilde{C}^{\prime}$ & $\mathrm{Du} \widetilde{C}^{\prime}$ \\
\hline c & $\mathrm{R}(2)$ & K (3) & $\begin{array}{l}\text { Pos (4) } \\
\text { Nec (5) }\end{array}$ \\
\hline $\mathrm{Cu} \widetilde{C}$ & & $\begin{array}{l}\mathrm{K}(6) \\
\mathrm{R}(7)\end{array}$ & $\begin{array}{l}\text { Pos (8) } \\
\mathrm{Nec}(9)\end{array}$ \\
\hline Du $\widetilde{C}$ & & & $\begin{array}{l}\text { Pos (10) } \\
\text { Nec (11) } \\
\text { Inc (15) } \\
\text { R (17) }\end{array}$ \\
\hline
\end{tabular}

crisp color whose membership degree to both fuzzy colors is at least $\alpha$ ) and, at the same time, their resemblance is 0 , meaning that both colors are semantically distinguishable and different. This is also consistent with the fact that the user considered both colors in his/her definition of the fuzzy color space since, conceptually, the fuzzy color space is a (fuzzy) partition of the crisp color space.

Hence, we separate the relationship between a crisp and a fuzzy color from the relationship between fuzzy colors. In other words, $S$ is not necessarily obtained by performing operations on the representations of the fuzzy colors. As a particular case, in this work we devote some effort to the study of the relation $S^{0}$ given by $S^{0}\left(\widetilde{C}, \widetilde{C^{\prime}}\right)=1$ iff $\widetilde{C}=\widetilde{C^{\prime}}$, and 0 otherwise. This is the simplest relation one may consider, and it is fully consistent with the view of $\widehat{X Y Z}$ as a partition of the set of crisp colors (though a fuzzy one in which colors may be compatible to some degree). The fact that under $S^{0}$ colors are all different but compatible illustrates very clearly the independence between fuzzy color definition and similarity. Other relations and how to obtain them for representing specific semantics will be studied in forthcoming papers.

The possible situations we may encounter when matching pieces of color information correspond to the cells in Table II, where c, c' $\in X Y Z$ are crisp colors, and $\widetilde{C}, \widetilde{C}^{\prime} \in \widetilde{X Y Z}$ are fuzzy colors. We shall denote $R=$ resemblance relation, $K=$ compatibility relation, $I n c=$ inclusion. $P o s$ and $N e c$ mean possibility and necessity, respectively.

A first notion of resemblance between crisp colors is that of resemblance induced by a single fuzzy color, defined as follows:

Definition 4.1: The resemblance between crisp colors induced by a single fuzzy color $\widetilde{C}$ is

$$
r_{\widetilde{C}}\left(c, c^{\prime}\right)=\min \left(\widetilde{C}(c), \widetilde{C}\left(c^{\prime}\right)\right)
$$

It is easy to show that $r_{\widetilde{C}}$ is symmetric and max-min transitive, but not reflexive unless $\widetilde{C}$ is crisp. Reflexivity may be imposed by forcing $r_{\widetilde{C}}(c, c)=1$ in the previous definition, allowing us to interpret directly a fuzzy color as a group of similar crisp colors, defined by a fuzzy equivalence relation. However, this introduces a discontinuity in the fuzzy set "being similar to $c$ following $\widetilde{C}$ ", whose membership function is given for every $c^{\prime}$ by $r_{\widetilde{C}}\left(c, c^{\prime}\right)$, even when $\widetilde{C}$ is continuous. This condition may be eliminated if continuity is desired and 
reflexivity is not mandatory. Anyway, this discussion is not very important from a practical point of view, since what is important in practical applications is the generalization of this notion to that of resemblance induced by a whole fuzzy color space, considering a user-defined resemblance relation $S$ between fuzzy colors, as follows:

Definition 4.2: The resemblance between crisp colors $c$ and $c^{\prime}$ induced by a fuzzy color space $\widehat{X Y Z}$ is

$R_{\widetilde{X Y Z}}\left(c, c^{\prime}\right)=\underset{\left(\widetilde{C}, \widetilde{C}^{\prime}\right) \in \widetilde{\max }}{\underset{X Y Y}{X Y Z}} \min \left\{\widetilde{C}(c), \widetilde{C}^{\prime}\left(c^{\prime}\right), S\left(\widetilde{C}, \widetilde{C}^{\prime}\right)\right\}$

Definition 4.2 is useful in practice when answering questions like hat RGB colors are resemblant to color $c$ ?, or Give me images containing colors that resemble that of pixel $p$, etc. Notice that in this case, the relation is reflexive iff the kernels of the fuzzy colors form a crisp partition of the crisp color space; otherwise, we have to impose $R_{\widetilde{X Y Z}}(c, c)=1$ and we have again a discontinuity in the similarity to a fixed crisp color $c$. Notice however that, taking into account the notion of fuzzy partition that we consider in Section II-B, we are allowed to consider a fuzzy color space consisting of non-crisp fuzzy subsets of colors whose kernels form a crisp partition of the color space, since we allow $\sum_{\widetilde{C} \in \widetilde{X Y Z}} \widetilde{C}(c)>1$. Hence, we may obtain a reflexive fuzzy relation $R_{\widetilde{X Y Z}}$ in which $R_{\widetilde{X Y Z}}\left(c, c^{\prime}\right)$ is continuous for every fixed $c$ (provided every $\widetilde{C} \in \widetilde{X Y Z}$ is continuous) from a suitable fuzzy color space $R_{\widetilde{X Y Z}}$ having non-crisp fuzzy colors.

The following definitions correspond to the rest of cases as shown in Table II. We shall assume from now on that we work with a fuzzy color space $\widehat{X Y Z}$, avoiding it explicitly in the notations. Notice that we shall always employ the same notation (letters $R$ and $K$ to denote resemblance and compatibility, respectively, etc.), although the definitions correspond to different resemblance and compatibility relations. Finally, we shall assume that only a subset of crisp colors in the color space $X Y Z$ are considered (this is always the case when working with computers).

Definition 4.3: The compatibility between a crisp color $c$ and a $\mathrm{Cu}$ fuzzy color $\widetilde{C} \in \overline{X Y Z}$ is

$$
K(c, \widetilde{C})=\max _{\widetilde{C}^{\prime} \in \overline{X Y Z}} \min \left\{\widetilde{C}(c), \widetilde{C}^{\prime}(c), S\left(\widetilde{C}, \widetilde{C}^{\prime}\right)\right\}
$$

Definition 4.3 is useful when answering questions like To what degree pixel p is red?, or Give me images containing red pixels, etc.

Definition 4.4: The possibility and the necessity that a certain color variable $V$ takes value $c$ knowing that its value is in a Du fuzzy color $\widetilde{C} \in \widehat{X Y Z}$ are, respectively

$$
\operatorname{pos}(V=c \mid V \text { is } \widetilde{C})=\widetilde{C}(c)
$$

and

$$
\operatorname{nec}(V=c \mid V \text { is } \widetilde{C})=1-\max _{c^{\prime} \in \widehat{X Y Z} \mid c^{\prime} \neq c}\left\{\widetilde{C}\left(c^{\prime}\right)\right\}
$$

Definition 4.4 is useful when answering questions like Knowing that my car is red, what is the possibility/necessity that my car is painted in the RGB color $c$ ?. Notice that for any fuzzy color $\widetilde{C} \in \widehat{X Y Z}$ such that its kernel is not a singleton, $\operatorname{Nec}(V=c \mid V$ is $\widetilde{C})=0$. On the other hand, if $\widetilde{C}$ is a singleton, it is easy to show that

$$
\begin{aligned}
\operatorname{pos}\left(V=c \mid V \text { is }\left\{c^{\prime}\right\}\right) & =\operatorname{Nec}\left(V=c \mid V \text { is }\left\{c^{\prime}\right\}\right)= \\
& = \begin{cases}1 & c=c^{\prime} \\
0 & \text { otherwise }\end{cases}
\end{aligned}
$$

Definition 4.5: The compatibility between two $\mathrm{Cu}$ fuzzy colors is

$$
\begin{aligned}
& K\left(\widetilde{C}, \widetilde{C^{\prime}}\right)=\max _{\left(\widetilde{C^{\prime \prime}}, \widetilde{C^{\prime \prime \prime}}\right) \in \widehat{X Y Z} \times \widetilde{X Y Z}} \max _{c \in X Y Z}\{ \\
& \left.\min \left\{S\left(\widetilde{C}, \widetilde{C^{\prime \prime}}\right), \widetilde{C^{\prime \prime}}(c), S\left(\widetilde{C^{\prime}}, \widetilde{C^{\prime \prime \prime}}\right), \widetilde{C^{\prime \prime \prime}}(c)\right\}\right\}
\end{aligned}
$$

Definition 4.5 is useful when answering questions like To what extent it is possible to find a crisp color that is red and orange?.

Definition 4.6: The resemblance between two $\mathrm{Cu}$ fuzzy colors is

$$
R\left(\widetilde{C}, \widetilde{C^{\prime}}\right)=S\left(\widetilde{C}, \widetilde{C^{\prime}}\right)
$$

where $S$ is a resemblance relation defined by the user.

Definition 4.6 is useful when answering questions like To what extent red is similar to orange?.

Definition 4.7: The possibility and the necessity that a certain color variable $V$ takes a value compatible with a $\mathrm{Cu}$ fuzzy color $\widetilde{C}$ knowing that the value of $V$ is in a Du fuzzy color $\widetilde{C^{\prime}}$ are, respectively

$$
\begin{gathered}
\operatorname{pos}\left(K(V, \widetilde{C}) \mid V \text { is } \widetilde{C^{\prime}}\right)= \\
=\max _{\widetilde{C^{\prime \prime}} \in \widetilde{X Y Z}} \max _{c \in X Y Z} \min \left\{\widetilde{C^{\prime}}(c), \widetilde{C^{\prime \prime}}(c), S\left(\widetilde{C}, \widetilde{C^{\prime \prime}}\right)\right\}
\end{gathered}
$$

and

$$
\begin{aligned}
& \operatorname{nec}\left(K(V, \widetilde{C}) \mid V \text { is } \widetilde{C^{\prime}}\right)=1-\operatorname{pos}\left(\neg K(V, \widetilde{C}) \mid V \text { is } \widetilde{C^{\prime}}\right)= \\
= & 1-\max _{\widetilde{C^{\prime \prime}} \in \widetilde{X Y Z}} \max _{c \in X Y Z} \min \left\{\widetilde{C^{\prime}}(c), 1-\widetilde{C^{\prime \prime}}(c), S\left(\widetilde{C}, \widetilde{C^{\prime \prime}}\right)\right\}
\end{aligned}
$$

where $\operatorname{pos}\left(\neg K(V, \widetilde{C}) \mid V\right.$ is $\left.\widetilde{C^{\prime}}\right)$ is the possibility that $V$ does not take a value compatible with $\widetilde{C}$ given that the value of $V$ is in $\widetilde{C^{\prime}}$.

Definition 4.7 is useful when answering questions like She told me the car was red, what is the possibility/necessity that the car was orange?

Definition 4.8: The possibility and the necessity that a certain color variable $V$ takes a value compatible with a Du fuzzy color $\widetilde{C}$ knowing that the value of $V$ is in a Du fuzzy color $\widetilde{C^{\prime}}$ are, respectively

$$
\begin{gathered}
\operatorname{pos}\left(K(V, \widetilde{C}) \mid V \text { is } \widetilde{C^{\prime}}\right)=\max _{c \in X Y Z} \min \left\{\widetilde{C^{\prime}}(c), \widetilde{C}(c)\right\} \\
n e c\left(K(V, \widetilde{C}) \mid V \text { is } \widetilde{C^{\prime}}\right)=1-\max _{c \in X Y Z} \min \left\{\widetilde{C^{\prime}}(c), 1-\widetilde{C}(c)\right\}
\end{gathered}
$$

Definition 4.8 is useful when answering questions like Jim saw a red car, and Tim saw an orange car, what's the 
possibility/necessity that both cars were painted in the same color?.

In order to define degrees of inclusion, we will employ the following definition from [17]:

Definition 4.9 ( [17]): Let $I$ a contrapositive fuzzy implication $^{1}$ satisfying:

1) $(\forall x, y \in[0,1])(x \leq y \Leftrightarrow I(x, y)=1)$

2) $(\forall x, y \in[0,1])(x=1 \wedge y=0 \Leftrightarrow I(x, y)=0)$

and let $A, B$ be two fuzzy sets defined on a crisp set $X$. Then, the family of functions given by

$$
\operatorname{Sub}(A, B)=\min _{x \in X} I(A(x), B(x))
$$

is a family of inclusion indicators that satisfy all the SinhaDougherty axioms [17].

An example of contrapositive fuzzy implication $I$ satisfying the conditions above is the Lukasiewicz's implicator [17], given by

$$
I_{a}(x, y)=\min (1,1-x+y)
$$

On this basis, a family of similarity indicators between fuzzy sets can be defined as follows [18]: let $A, B$ be two fuzzy sets defined on a crisp set $X$. Then, the family of functions given by

$$
\operatorname{Sim}(A, B)=\min \{\operatorname{Sub}(A, B), \operatorname{Sub}(B, A)\}
$$

is a family of similarity indicators.

The inclusion indicator (resp. similarity indicator) between pieces of color information is useful when answering questions like To what extent Jim's knowledge about my car's color is more specific than (resp. similar to) Tim's?.

Definition 4.10: The degree of inclusion of a Du fuzzy color $\widetilde{C}$ in another Du fuzzy color $\widetilde{C}^{\prime}$ is calculated by means of a suitable, application-depending inclusion indicator in the family given by Eq. 12, i.e.,

$$
\operatorname{Inc}\left(\widetilde{C}, \widetilde{C^{\prime}}\right)=\operatorname{Sub}\left(\widetilde{C}, \widetilde{C^{\prime}}\right)=\min _{c \in X Y Z} I\left(\widetilde{C}(c), \widetilde{C^{\prime}}(c)\right)
$$

In particular, the degree of inclusion of a Du fuzzy color $\widetilde{C}$ in another Du fuzzy color $\widetilde{C^{\prime}}$ based on Lukasiewicz's implicator is

$$
\operatorname{Inc}\left(\widetilde{C}, \widetilde{C^{\prime}}\right)=\min _{c \in X Y Z} \min \left(1,1-\widetilde{C}(c)+\widetilde{C^{\prime}}(c)\right)
$$

Definition 4.11: The degree of resemblance between two Du fuzzy colors $\widetilde{C}$ and $\widetilde{C}^{\prime}$ is calculated by means of one function of the family $\mathrm{Sim}$ given by Eq. 14, i.e.,

$$
R\left(\widetilde{C}, \widetilde{C^{\prime}}\right)=\operatorname{Sim}\left(\widetilde{C}, \widetilde{C^{\prime}}\right)
$$

${ }^{1}$ A fuzzy implication $I$ is contrapositive if $I(x, y)=I\left(N_{I}(y), N_{I}(x)\right)$

\begin{tabular}{|c|c|c|c|}
\hline & c' & $\mathrm{Cu} \widetilde{C}^{\prime}$ & Du $\widetilde{C}^{\prime}$ \\
\hline \multirow[t]{2}{*}{ c } & R (18) & K (19) & Pos (4) \\
\hline & & & $\operatorname{Nec}(5)$ \\
\hline \multirow[t]{2}{*}{$\overline{\mathrm{Cu} \widetilde{C}}$} & & K (20) & Pos (22) \\
\hline & & $\mathrm{R}(21)$ & $\mathrm{Nec}$ (23) \\
\hline \multirow[t]{4}{*}{ Du $\widetilde{C}$} & & & Pos (10) \\
\hline & & & $\mathrm{Nec}(11)$ \\
\hline & & & Inc (15) \\
\hline & & & R (17) \\
\hline
\end{tabular}
$\forall x, y \in[0,1]$, with $N_{I}$ being the induced negator of $I$ defined as $N_{I}(x)=$ $I(x, 0) \forall x \in[0,1]$.
TABLE III

MATCHING PIECES OF COLOR INFORMATION USING $S^{0}$, AND THE CORRESPONDING EQUATION NUMBER.

\section{THE CASE OF THE RESEMBLANCE RELATION $S^{0}$}

In this section we consider the particular case of the relation $S^{0}$ given by $S^{0}\left(\widetilde{C}, \widetilde{C^{\prime}}\right)=1$ iff $\widetilde{C}=\widetilde{C^{\prime}}$, and 0 otherwise. As explained in the previous section, the resemblance relation $S$ between fuzzy colors is not necessarily obtained from the membership functions of the fuzzy colors. In principle, we assume there is independence between both these aspects, i.e., they are two different types of information to be provided by humans. Whilst membership functions define the compatibility between crisp and fuzzy colors, the resemblance relation $S$ define the semantic resemblance between fuzzy colors, that depend on how a particular use perceive and use them.

Since a fuzzy color space is defined as a partition of a crisp color space using fuzzy colors, it is reasonable to consider that these fuzzy colors are the different groups that may be distinguished and separated. The fact that the partition is fuzzy allows fuzzy colors to be compatible, i.e., to share crisp colors in their support, but this is not incompatible with the view that colors in $\widehat{X Y Z}$ are totally different.

This consideration is useful for building a fuzzy color space by asking users to enumerate the collection of different colors they can clearly distinguish as different. Hence, $S^{0}$ is the simplest relation between fuzzy colors and, at the same time, the less computationally complex. In [12] we proposed an approach for defining a fuzzy color space from a collection of color names and a corresponding set of representative crisp colors, one for each name, both sets provided by the user. If the user is asked to provide these sets by assuming $S^{0}$ as the resemblance between fuzzy colors, we have in a very simple way all the necessary information for defining a fuzzy color space and for using this space for representing and matching color information for different purposes.

Using $S^{0}$, the relations studied in section IV reduce to those referred in Table III. Some expressions (those not involving the similarity between fuzzy colors $S$ ) are the same. New formulae are given in equations (18) - (23).

$$
\begin{aligned}
R_{\widetilde{X Y Z}}\left(c, c^{\prime}\right) & =\max _{\widetilde{C} \in \widetilde{X Y Z}} \min \left\{\widetilde{C}(c), \widetilde{C}\left(c^{\prime}\right)\right\} \\
K(c, \widetilde{C}) & =\widetilde{C}(c) \\
K\left(\widetilde{C}, \widetilde{C^{\prime}}\right) & =\max _{c \in X Y Z} \min \left\{\widetilde{C}(c), \widetilde{C^{\prime}}(c)\right\} \\
R\left(\widetilde{C}, \widetilde{C^{\prime}}\right) & =1 \text { if } C=C^{\prime}, 0 \text { otherwise. }
\end{aligned}
$$




$$
\begin{aligned}
\operatorname{pos}\left(K(V, \widetilde{C}) \mid V \text { is } \widetilde{C^{\prime}}\right) & =\max _{c \in X Y Z} \min \left\{\widetilde{C}(c), \widetilde{C^{\prime}}(c)\right\} \\
\operatorname{nec}\left(K(V, \widetilde{C}) \mid V \text { is } \widetilde{C^{\prime}}\right) & =1-\operatorname{pos}\left(\neg K(V, \widetilde{C}) \mid V \text { is } \widetilde{C^{\prime}}\right)= \\
= & 1-\max _{c \in X Y Z} \min \left\{\widetilde{C^{\prime}}(c), 1-\widetilde{C}(c)\right\}
\end{aligned}
$$

\section{CONCLUSIONS AND FUTURE WORK}

Our work lies in the framework of Zadeh's Computational Theory of Perceptions [19]. Fuzzy color spaces are intended to represent the collection of terms employed by a particular user, either in general or in a certain application, for describing his/her perception about colors. By representation we understand the (fuzzy) compatibility between crisp colors and these terms, expressed as membership functions defined on a crisp color space. In addition, we consider separately the user's perception of resemblance between colors, the latter seen as abstract notions, by means of a fuzzy relation $S$. These models allow us to fill the semantic gap between the representation of colors in computers and the perception of colors by humans, and to represent different types of color information. As a consequence, we are able to provide computational procedures for matching color information of different types, something very useful in determining the compatibility between linguistic terms and images for color image description, for image and visual information retrieval, flexible query answering in image databases, etc.

As future work, we shall use the relations introduced here in many applications, in particular for developing image retrieval systems working with linguistic queries and flexible queries to image databases, as well as for identifying visual concepts and the automatic generation of linguistic descriptions of images on the basis of color information [20]. We will also employ these techniques for color image segmentation [21], [22]. Another important future work concerns the study of the computational complexity of calculating the measures proposed in this work, when it is possible to precalculate this information, and how to develop efficient algorithms for using them in practice. Finally, we shall study the semantics of different similarity relations between fuzzy colors, in particular on the basis of hierarchical fuzzy color spaces, a notion that we are currently developing.

\section{REFERENCES}

[1] A. Plouznikoff, N. Plouznikoff, J. Robert, and M. Desmarais, "Enhancing human-machine interactions: virtual interface alteration through wearable computers," in CHI '06: Proceedings of the SIGCHI conference on
Human Factors in computing systems. New York, NY, USA: ACM, 2006, pp. 373-376.

[2] P. Kay and C. McDaniel, "The linguistic significance of the meanings of soft basic color terms," anguage, vol. 3, no. 54, pp. 610-646, 1978.

[3] J. Lammens, "A computational model of color perception and color naming," State University of New York, Tech. Rep. 94-26, 24, 1994.

[4] N. Sugano, "Color-naming system using fuzzy set theorical approach," in IEEE Int. Conference on Fuzzy Systems, 2001, pp. 81-84.

[5] - "Fuzzy set theoretical approach to the RGB color triangle," in Knowledge-Based Intelligent Information and Engineering Systems. NCS 4253. Springer-Verlag, 2006, pp. 948-955.

[6] H. Zhu, H. Zhang, and Y. Yu, "Deep into color names: Matching color descriptions by their fuzzy semantics," NAI 4183, pp. 138-149, 2006.

[7] A. Mojsilovic, "A computational model for color naming and describing color composition of images," IEEE Transactions on Image Processing, vol. 14, no. 5, pp. $690-699,2005$.

[8] A. Younes, I. Truck, and H. Akdag, "Color image profiling using fuzzy sets," Turkish Journal of Electrical Engineering and Computer Sciences, vol. 13 , no. 3, p. 343359, 2005.

[9] J. Chamorro-Martínez, J. Medina, C. Barranco, E. Galán-Perales, and J. Soto-Hidalgo, "Retrieving images in fuzzy object-relational databases using dominant color descriptors," Fuzzy Sets and Systems, vol. 158, no. 3, pp. $312-324,2007$.

[10] H. Aboulmagd, N. El-Gayar, and H. Onsi, "A new approach in contentbased image retrieval using fuzzy," Telecommunication Systems, vol. 40, no. 2, pp. 55-66, 2009.

[11] R. Benavente, M. Vanrell, and R. Baldrich, "Parametric fuzzy sets for automatic color naming," Journal of the Optical Society of America $A$, vol. 25 , no. 10 , pp. 2582-2593, Oct 2008. [Online]. Available: http://cat.uab.cat/Publications/2008/BVB08

[12] J. Soto-Hidalgo, J. Chamorro-Martínez, and D. Sánchez, "A new approach for defining a fuzzy color space," in Proceedings CCI 2010, 2010, pp. 292-297.

[13] L. Zadeh, "PRUF - a meaning representation language for natural languages," International Journal of Man-Machine Studies, vol. 10, pp. 395-460, 1978.

[14] R. Yager, "Set-based representations of conjunctive and disjunctive knowledge," Information Sciences, vol. 41, no. 1, pp. 1-22, 1987.

[15] D. Dubois and H. Prade, "Incomplete conjunctive information," Computers \& mathematics with applications, vol. 15, pp. 797-810, 1988.

[16] _ , "Gradualness, uncertainty and bipolarity: Making sense of fuzzy sets," Fuzzy Sets and Systems, vol. In Press, Corrected Proof, 2010.

[17] C. Cornelis, C. Van der Donck, and E. Kerre, "Sinha-Dougherty approach to the fuzzification of set inclusion revisited," Fuzzy Sets and Systems, vol. 134, pp. 283-295, 2003.

[18] I. Beg and S. Ashraf, "Similarity measures for fuzzy sets," Appl. and Comput. math., vol. 8, no. 2, pp. 192-202, 2009.

[19] L. Zadeh, "A new direction in AI. Toward a computational theory of perceptions," AI Magazine, vol. 22, no. 1, pp. 73-84, 2001.

[20] R. Castillo-Ortega, J. Chamorro-Martínez, N. Marín, D. Sánchez, and J. Soto-Hidalgo, "Describing images via linguistic features and hierarchical segmentation," in Proceedings CCI 2010, 2010, pp. 1104-1111.

[21] J. Chamorro-Martínez, D. Sánchez, B. Prados-Suarez, and E. GalánPerales, "Fuzzy connectivity measures for path-based image segmentation," in IEEE International Conference on Fuzzy Systems. Reno, Nevada (USA), May 2005, pp. 218-223.

[22] B. Prados-Suárez, J. Chamorro-Martínez, D. Sánchez, and J. Abad, "Region-based fit of color homogeneity measures for fuzzy image segmentation," Fuzzy Sets and Systems, vol. 158, no. 3, pp. 215-229, 2007 